\title{
The Deep Lymphatic Anatomy of the Foot
}

\author{
Rui Ma ${ }^{1}$, Chuan Xiang Ma ${ }^{1}$, Wei Ren Pan ${ }^{1 *}$, Fan Qiang Zeng ${ }^{1}$, Zhi Qiang Qiu ${ }^{2}$, Mei Ying Liu ${ }^{1}$ and Zhi An Liu ${ }^{1}$ \\ ${ }^{1}$ Department of Anatomy, College of Biomedical Sciences, Xuzhou Medical University, China \\ ${ }^{2}$ Department of Radiology, Xuzhou Oriental People’s Hospital, Xuzhou Medical University, China
}

\begin{abstract}
Background: Detail of the deep lymphatic anatomy of the foot remains the least described in medical literature.

Methods: Eight feet were harvested from four unembalmed human cadavers amputating above the ankle. A small amount of $6 \%$ hydrogen peroxide was used to find the deep lymphatic vessels around the dorsal and plantar metatarsal vessels. A fine needle was inserted into vessels and injected with a barium sulphate compound. Each specimen was dissected, photographed, and radiographed to demonstrate the deep lymphatic distribution of the foot.

Results: Deep collecting lymph vessels of the foot were found in the deep tissue of the dorsal and sole of the foot, they were: Dorsal metatarsal lymph vessel (DMLV); Lateral tarsal lymph vessel (LTLV); Central tarsal lymph vessel (CTLV); Medial tarsal lymph vessel (MTLV); Dorsalis pedis lymph vessel (DPLV); Anterior lateral malleolus lymph vessel (ALMLV); Plantar metatarsal lymph vessel (PMLV); Deep plantar arch lymph vessel (DPALV); Lateral plantar lymph vessel (LPLV); Medial plantar lymph vessel (MPLV); Medial calcaneus lymph vessel (MCLV); Medial malleolus perforating lymph vessel (MMPLV). Each vessel ran concentrically, then merged successively and finally drained into the anterior or posterior tibial lymph vessels.
\end{abstract}

Conclusion: The deep lymphatic distribution of the foot has been presented. The results will provide an anatomical basis for clinical management, educational reference, and scientific research.

KEYWORDS: Foot; Deep lymphatic vessel; Perforating lymph vessel; Vascularised lymph vessel flap

ABBREVIATIONS: DMLV: Dorsal Metatarsal Lymph Vessel; LTLV: Lateral Tarsal Lymph Vessel; CTLV: Central Tarsal Lymph Vessel; MTLV: Medial Tarsal Lymph Vessel; DPLV: Dorsalis Pedis Lymph Vessel; ALMLV: Anterior Lateral Malleolus Lymph Vessel; PMLV: Plantar Metatarsal Lymph Vessel; DPALV: Deep Plantar Arch Lymph Vessel; LPLV: Lateral Plantar Lymph Vessel; MPLV: Medial Plantar Lymph Vessel; MCLV: Medial Calcaneus Lymph Vessel; MMPLV: Medial Malleolus Perforating Lymph Vessel

\section{INTRODUCTION}

The lymphatic system plays an important role in human health and disease. Clinical management requires accurate and detailed anatomical knowledge of the human lymphatic system. Although, the superficial and deep lymphatic anatomy in the lower limb has been updated during the last decade by using the new technique [1-4], but the deep lymphatic anatomy remained in uncertainty. Therefore, further study should be carried to renew the knowledge of the human lymphatic system to meet the needs of precise clinical management, modern education, and advanced scientific research. In this study, details of the deep lymphatic distribution and the communicating lymph vessel in the foot are described and demonstrated by radiographs and photographs.

\section{MATERIALS AND METHODS}

The investigation was performed with appropriate institutional ethics approval. For this study, a total of 8 feet were harvested from 4 unembalmed human cadavers, disarticulated $10 \mathrm{~cm}$ above the ankle joint. There were 2 males and 2 females aged 70 to 80 years old (average age 74 years old). A small amount of $6 \%$ hydrogen peroxide (Zhonglian Chemical Co., Ltd, Suzhou, China), injected with a $1 \mathrm{ml}$ syringe, was injected into the tissue around the dorsal and plantar metatarsal vessels, medial and lateral tarsal vessels, arcuate vessel, deep planter arch, medial and lateral plantar vessels, anterior medial and lateral malleolar vessels in respective order. Under a surgical microscope (Leica Microsystems Ltd, Heerbrugg,

\begin{tabular}{|c|c|}
\hline & $\begin{array}{l}\text { Address for correspondence: Wei-Ren Pan and Zhi An Liu Department of Anatomy, } \\
\text { College of Biomedical Sciences, Xuzhou Medical University, China }\end{array}$ \\
\hline & $\begin{array}{l}\text { Received: March 03, } 2021 \quad \text { Published: March 19, } 2021 \\
\text { How to cite this article: Rui M, Chuan XM, Wei RP, Fan QZ, Zhi QQ, Mei YL, Zhi AL. The Deep } \\
\text { Lymphatic Anatomy of the Foot. 2021- 3(2) OAJBS.ID.000266. DOI: 10.38125/OAJBS.000266 }\end{array}$ \\
\hline
\end{tabular}


Switerland) the distended lymphatic vessels were identified in the area. Each vessel was inserted by a fine glass needle or 30-gauge needle (Zhejiang KDL Medical Equipment Group Ltd., Wenzhou, China; Precision glide needle: Becton Dickinson \& Co, Franklin Lakes, N.J., USA) and injected with a radio-opaque mixture (Barium Sulphate 15g: Shanghai Silian Indusry Co. Ltd., China; Milk powder 5g: Heinz Ltd., Qingdao, China; Concentrated poster color - dark green 3g: Liaoyuan arts and stationery Ltd., Hunang, China; Water $20 \mathrm{ml}$ ). Lymphatic vessels were then traced, photographed, and radiographed (Digital X-ray Dignostic System - Multix Select DR: Siemens Healthcare Diagnostic Co. Ltd., Erlangen, Germany) to demonstrate their distribution. The results were transferred to computer (Dell Vostro 200: Dell Computer Inc. Chinese Division, P.R. China) for image analysis using Photoshop software (Adobe Photoshop CS5 V12, Adobe Systems Software Co., Ltd, Beijing, China).

\section{RESULTS}

Deep collecting lymph vessels of the foot were found in the deep tissue of the dorsal and sole of the foot. Each vessel ran concentrically, some of them merged, and then drained into the anterior or posterior lymph vessels.

\section{Deep Collecting Lymph Vessels on the Dorsal Foot}

Dorsal metatarsal lymph vessels (DMLV): Above each dorsal interosseous muscle, the $1^{\text {st }}$ to $4^{\text {th }}$ DMLVs arose respectively near the metatarsal head and ran concentrically accompanying corresponding blood vessels. They travelled near the metatarsal base, passed through the interosseous spaces, and then merged into DPALV (Figure 1\& 2). The average diameter of the vessel was $0.2 \mathrm{~mm}$ (ranging from 0.1 to $0.3 \mathrm{~mm}$ ).

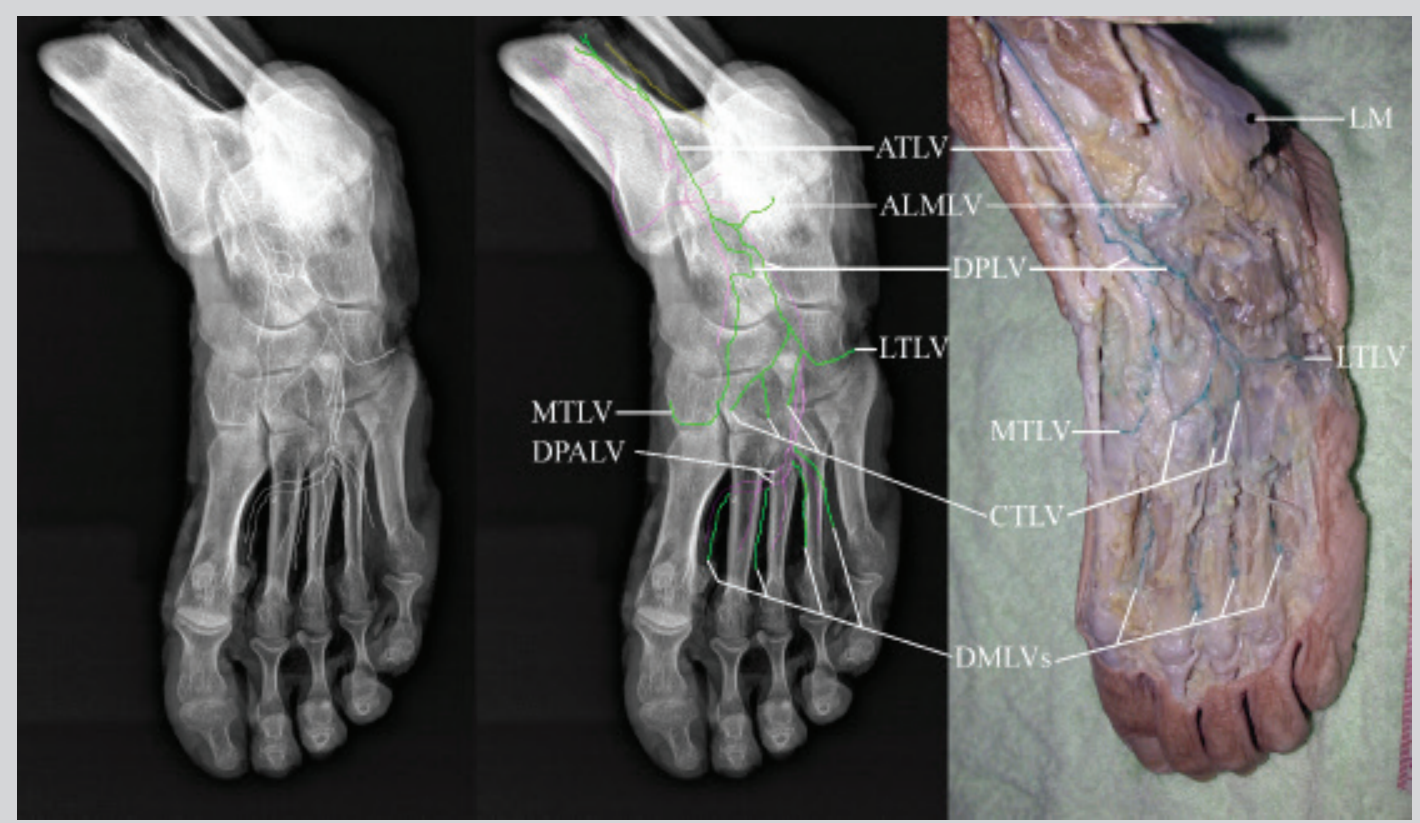

Figure 1: Distribution of the deep lymphatics in the left foot. Left and center images: radiographs, AP view; right photograph: dorsal view.

Lateral Tarsal Lymph Vessel (LTLV): Arising from the midlateral edge of the foot, LTLV travelled upwards and then obliquely towards the dorsal foot and continued its course as DPLV (Figure 1 \& 2). During the course, it received 1-2 lymph vessels arising from the centre of the dorsal foot. The average diameter of the vessel was $0.3 \mathrm{~mm}$ (ranging from 0.2 to $0.4 \mathrm{~mm}$ ).

Central Tarsal Lymph Vessel (CTLV): Arising around the arcuate blood vessel, 2 to 3 CTLVs travelled obliquely towards LTLV, and then formed 2 vessels to converge with LTLV (Figure $1 \& 2$ ). The average diameter of the vessel was $0.3 \mathrm{~mm}$ (ranging from 0.2 to 0.4 $\mathrm{mm})$.

Medial Tarsal Lymph Vessel (MTLV): Arising from the midmedial edge of the foot, MTLV travelled upwards and then obliquely towards the dorsal foot and continued its course as DPLV (Figure 1 $\& 2$ ). The average diameter of the vessel was $0.3 \mathrm{~mm}$ (ranging from 0.2 to $0.4 \mathrm{~mm}$ ).

Anterior Lateral Malleolus Lymph Vessel (ALMLV): Arising from the anterior aspect of the lateral malleolus (LM), ALMLV travelled forward-obliquely to the anterior aspect of the ankle joint and then entered DPLV (Figure $1 \& 2$ ). The average diameter of the vessel was $0.25 \mathrm{~mm}$ (ranging from 0.2 to $0.3 \mathrm{~mm}$ ).

Dorsalis Pedis Lymph Vessel (DPLV): Continuing from LTLV and MTLV, two DPLVs traveled on each side of corresponding blood vessels, converged at the anterior aspect of the ankle joint and then continued its course as the anterior tibial lymph vessel (ATLV) running with their respective blood vessels (Figure $1 \& 2$ ). The average diameter of the vessel was $0.35 \mathrm{~mm}$ (ranging from 0.3 to $0.4 \mathrm{~mm}$ ).

\section{Deep Collecting Lymph Vessels in The Plantar}

Plantar Metatarsal Lymph Vessel (PMLV): Below the deep surface of dorsal/plantar interosseous muscle, the $1^{\text {st }}$ to $4^{\text {th }}$ PMLVs arose respectively near the metatarsal head and ran concentrically with corresponding blood vessels. They merged into DPALV near the metatarsal base (Figure 3). The average diameter of the vessel was $0.2 \mathrm{~mm}$ (ranging from 0.1 to $0.3 \mathrm{~mm}$ ).

Deep Plantar Arch Lymph Vessel (DPALV): Two DPALVs were found running with corresponding blood vessels (Figure 3). 
One situated slightly deep-anteriorly that received the $1^{\text {st }}$ to $4^{\text {th }}$ DMLVs (Figure 1 \& 3), the other one slightly superficial-posteriorly that received the $1^{\text {st }}$ to $4^{\text {th }}$ PMLVs (Figure 3). Two DPALVs ran posterolaterally to form the LPLV at the mid-lateral plantar and then converged to one vessel (Figure $2 \& 4$ ). The average diameter of the vessel was $0.3 \mathrm{~mm}$ (ranging from 0.2 to $0.4 \mathrm{~mm}$ ).
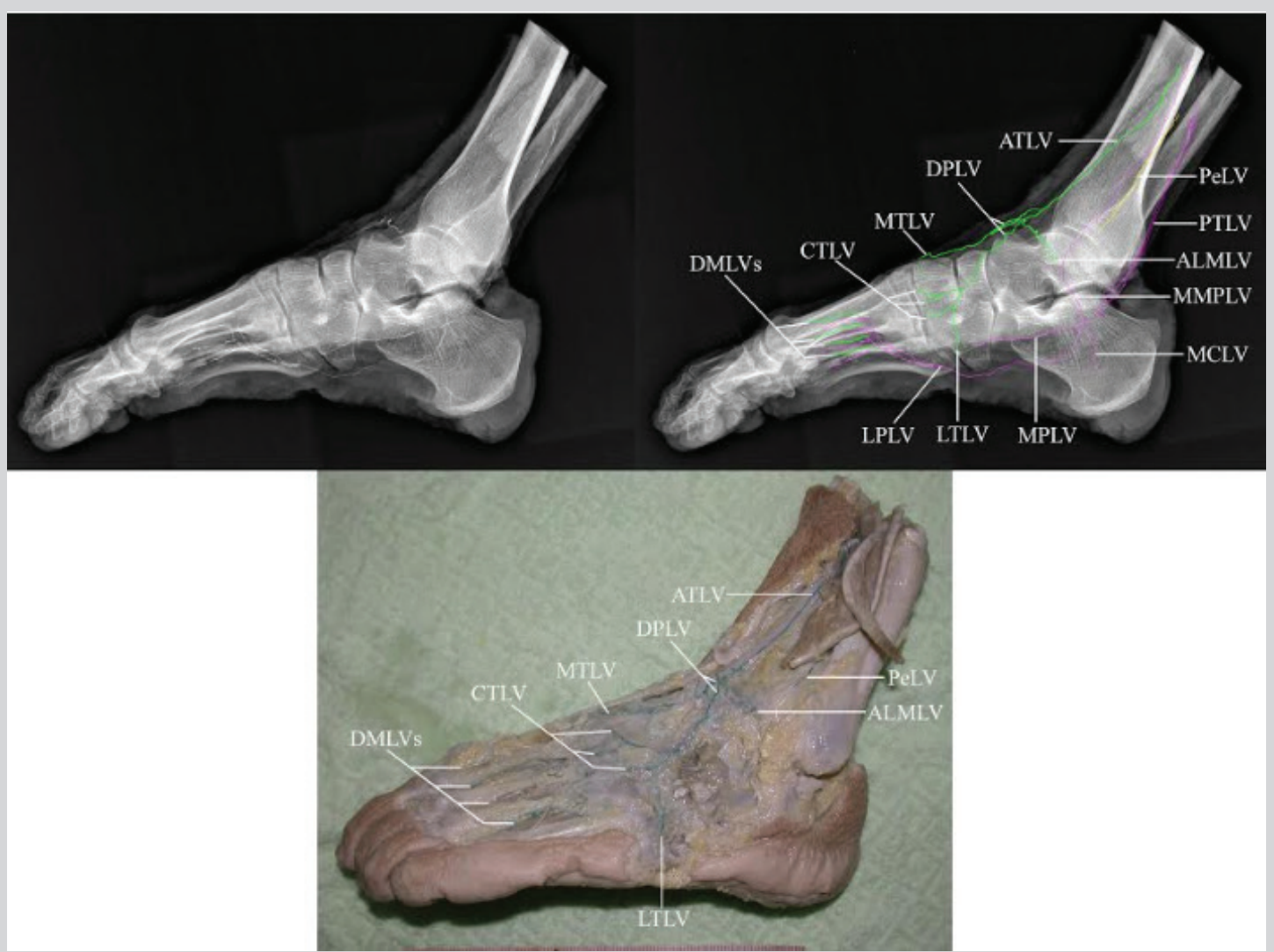

Figure 2: Distribution of the deep lymphatics in the left foot. Above images: radiographs, lateral view; below photograph: lateral view.

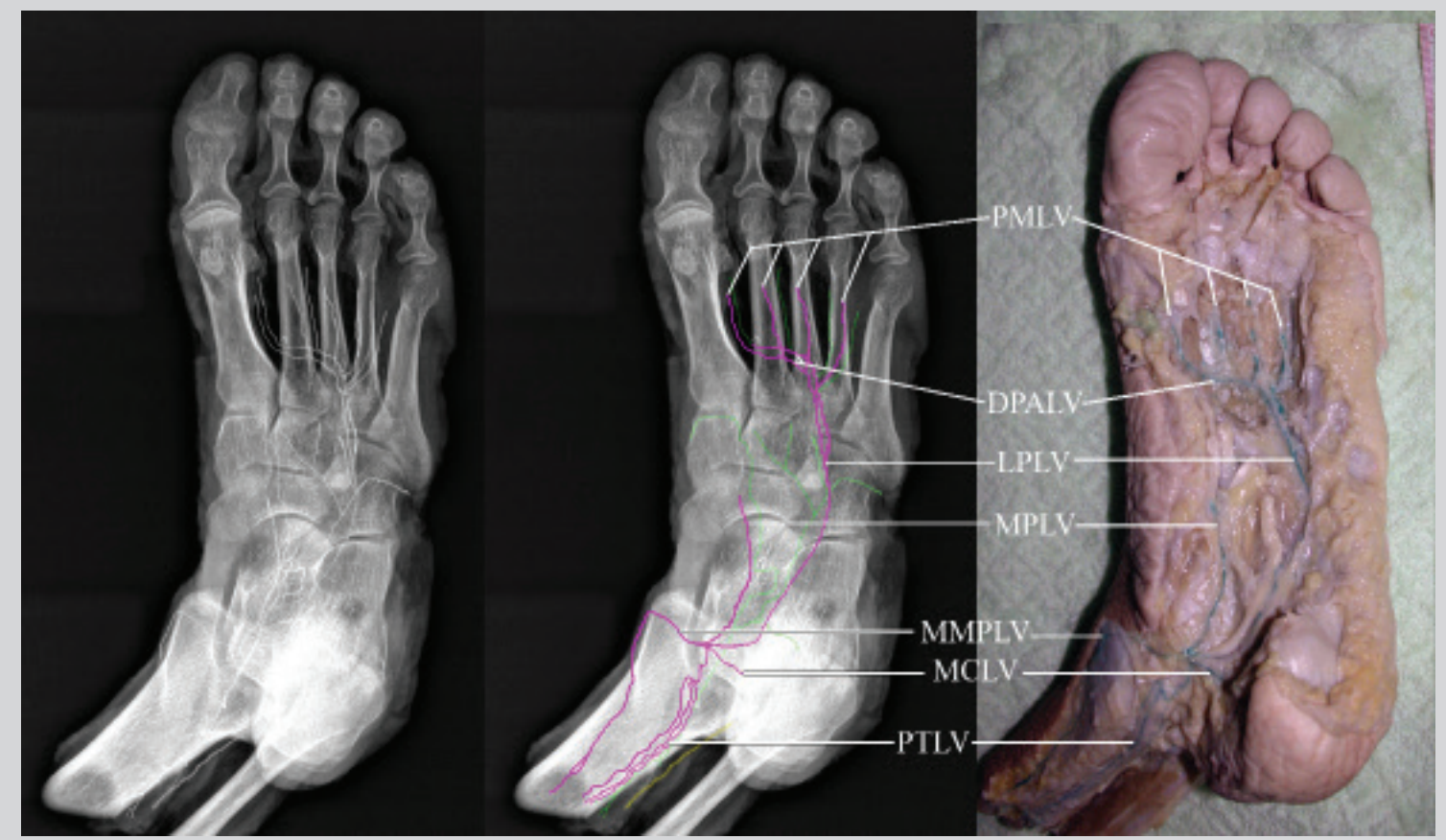

Figure 3: Distribution of the deep lymphatics in the left foot. Left and center images: radiographs, PA view; right photograph: plantar view. 


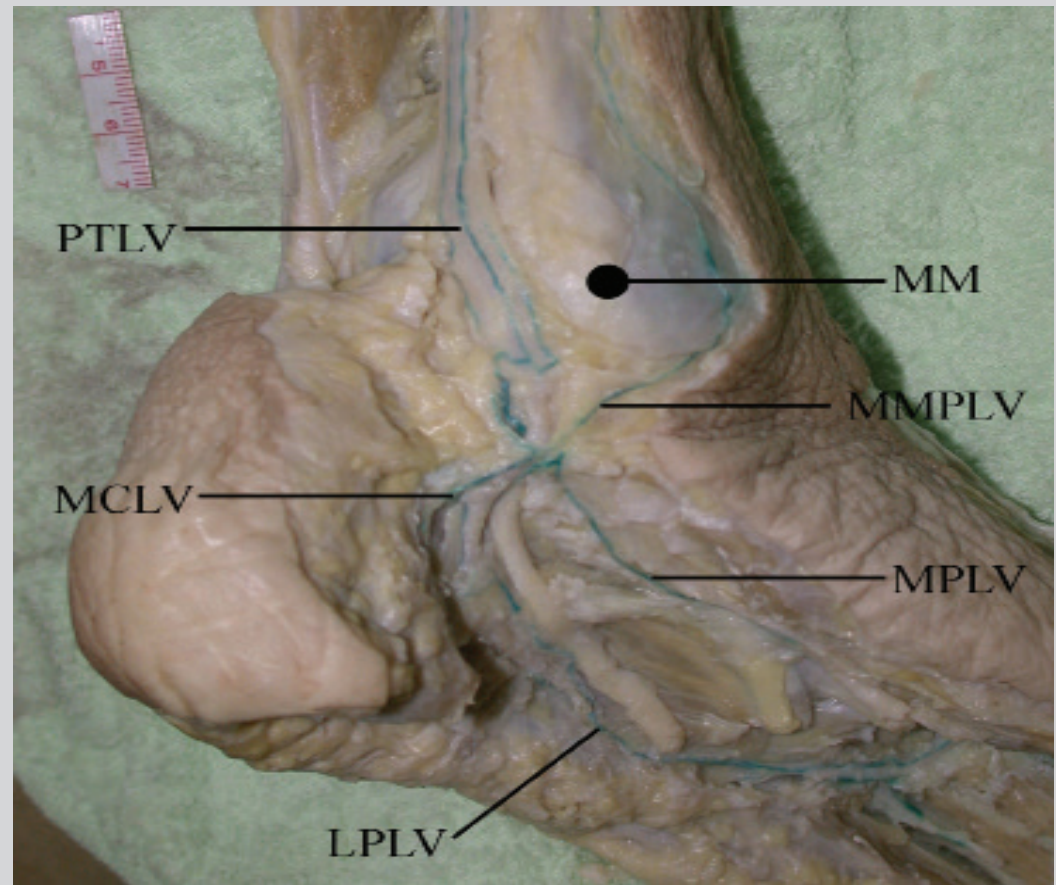

Figure 4: Distribution of the deep lymphatics around the medial malleolus of the left foot.

Lateral Plantar Lymph Vessel (LPLV): Continuing from DPALV, two LPLVs traveled on the lateral side of the plantar with corresponding blood vessels and converged to one vessel around the mid-lateral plantar (Figure 2 \& 3). Continuing the course posteriorly, it gradually turned medially to converge with MPLV and MCLV at the bifurcation of the lateral and medial plantar arteries, and then continued its course as the posterior tibial lymph vessel (PTLV) (Figure $2 \& 4$ ). The average diameter of the vessel was 0.35 $\mathrm{mm}$ (ranging from 0.3 to $0.4 \mathrm{~mm}$ ).

Medial Plantar Lymph Vessel (MPLV): Arising from the proximal third of the medial plantar, MPLV travelled posteriorly with corresponding blood vessels, converged with PTLV and MCLV at the bifurcation of the lateral and medial plantar arteries, and then continued its course as the posterior tibial lymph vessel (PTLV) (Figure $2 \& 4$ ). The average diameter of the vessel was 0.3 $\mathrm{mm}$ (ranging from 0.2 to $0.4 \mathrm{~mm}$ ).

Medial Calcaneus Lymph Vessel (MCLV): Arising from the lateral aspect of the heel, MCLV travelled obliquely with corresponding blood vessels and then converged with MPLV and PTLV at the bifurcation of the lateral and medial plantar arteries, (Figure $2 \& 4$ ). The average diameter of the vessel was $0.3 \mathrm{~mm}$ (ranging from 0.2 to $0.4 \mathrm{~mm}$ ).

Medial Malleolus Perforating Lymph Vessel (MMPLV): In one specimen, a MMPLV arising from the confluence of LPLV and MCLV travelled up forward-obliquely, bypassed the medial malleolus (MM) to the anterior aspect of the tibia and then ran upwards in the subcutaneous of the anterior aspect of the tibia as a superficial collecting lymph vessel (Figure $3 \& 4$ ). The average diameter of the vessel was $0.3 \mathrm{~mm}$ (ranging from 0.2 to $0.4 \mathrm{~mm}$ ).

\section{DISCUSSION}

Recalling the history of research conducted on the lymphatic system, it showed that the lymphatic anatomy of limbs was not available until the mercury infusion technique was applied [1-4]. Although the knowledge of the superficial and deep lymphatic anatomy of limbs has been updated during the last decade [5-8] the deep lymphatic anatomy of the foot remains the least described in medical literature. In this study, we have presented the deep lymphatic distribution of the foot. The characteristics of the drainage pattern are described as follows.

a) They arose from the different areas and deep layers in the dorsal and plantar.

b) Most dorsal lymph vessels drained in different directions first and then all turned and ran towards the DPLV.

c) Either DMLV or PMLV merged into DPALV.

d) LPLV, MPLV and MCLV converged in the bifurcation of the lateral and medial plantar arteries, and then continued its course as the posterior tibial lymph vessel.

e) Occasionally a MMPLV, arising from the confluence of LPLV and MCLV, travelled up forward-obliquely to the anterior aspect of the tibia and then ran upwards in the subcutaneous of the anterior aspect of the tibia as a superficial collecting lymph vessel.

The iatrogenic lymphoedema of the upper limb is an unsolved complication after breast cancer surgery. With a progressive pathologic condition, there is interstitial accumulation of proteinrich lymphatic fluid, recurrent cellulitis, adipose tissue hypertrophy and fibrosis resulting in an increase of volume and chronic edema in the affected region. Several surgical procedures have been designed for reducing the edema in the affected extremity.

A vascularized lymph vessel flap transfer was suggested for treating obstructive lymphedema by bridging the site of the obstruction to divert the lymph flow [9-11]. Except for the groin and forearm the dorsal foot is also a potential donor site for harvesting the flap (Figure 5). If the full thickness of the skin, as a vascularized lymph vessel flap, on the dorsal foot with the superficial lymphatic vessels [2] was harvested, the deep collecting vessels would still perform their function. 


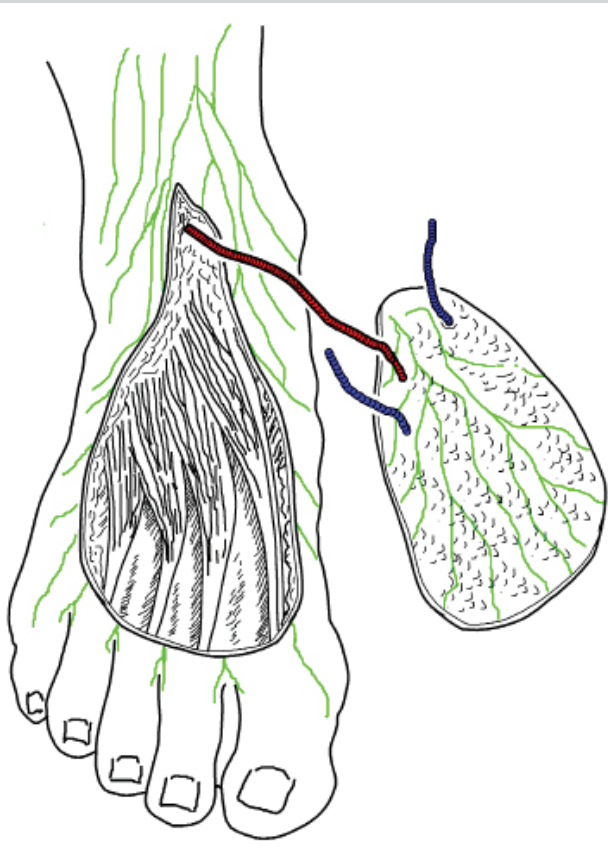

Figure 5: Sketches of potential vascularized lymph vessel flap on the dorsal foot.

\section{CONCLUSION}

The deep lymphatic drainage of the foot has been described. The result will provide an anatomical basis for clinical management, educational reference, and scientific research.

\section{ACKNOWLEDGEMENT}

Authors would like to pay tribute to body donors who contributed their bodies for this study and thank their families for their assistance. Many thanks to the National Natural Science Foundation of China (No: 31671253), Xuzhou Medical University President special fund (No: 53051116) and the foreign experts special fund of Department of International Cooperation and Exchange (No: 537101) for supporting this study.

\section{REFERENCES}

1. Pan WR, Le RCM, Levy SM (2011) Alternative lymphatic drainage routes from the lateral heel to the inguinal lymph nodes: Anatomic study and clinical implications. ANZ J Surg 81: 431-435.

2. Pan WR, Levy SM, Wang DG, Chen Y (2013) Superficial lymphatic drainage of the lower extremity: anatomical study and clinical implication. Plast Recon Surg 132: 696-707.
3. Pan WR, Levy SM, Wang DG, Chen Y (2016) Radiologic manifestations of senile lymph nodes in the popliteal fossa. Lymphat Res Bio 14: 25-29.

4. Pan WR, Zeng FQ, Wang DG, Qiu ZQ (2017) Perforating and deep lymphatic vessels in the knee region: an anatomical study and clinical implications. ANZ J Surg 87(5): 404-410.

5. Bonamy C, Broca P, Beau ME (1844) Atlas danatomie descriptive du corps humain. Paris, Fotin, Masson.

6. Sappey PC (1874) Anatomie, physiologie, pathologie des vaisseaux lymphatiques. Paris: Adrien Delahaye.

7. Pan WR (2017) Atlas of lymphatic anatomy in the head, neck, chest and limbs. Beijing: People's Medical publishing house, Singapore.

8. Ma CX, Pan WR, Liu ZA, Zeng FQ, Qiu ZQ, et al. (2018) The deep lymphatic anatomy of the hand. Ann Anat 218: 105-109.

9. Greene AK, Slavin SA, Brorson H (2015) Lymphedema: presentation, diagnosis, and treatment. New York, USA.

10. Taylor GI, Pan WR (2014) The Angiosome concept and tissue transfer. Quality Medical Publishing, St. Louis, USA.

11. Chen WF, Nurlen M MC, Ding J, Bowem M (2019) Vascularized lymph vessel transfer for extremity lymphedema - is transfer of lymph node still necessary? Int Microsurg J 3(1): 1-7. 